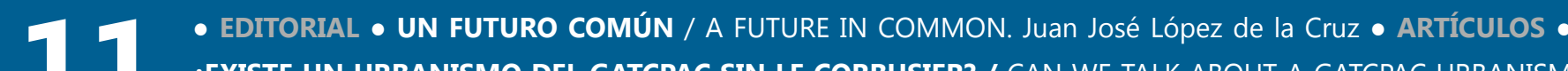
¿EXISTE UN URBANISMO DEL GATCPAC SIN LE CORBUSIER? / CAN WE TALK ABOUT A GATCPAC URBANISM WITHOUT LE CORBUSIER'S INFLUENCE? Roger Joan Sauquet Llonch • ODAM - A CONSTRUÇÃO DO MODERNO EM PORTUGAL: ENTRE O UNIVERSAL E O SINGULAR / ODAM - THE CONSTRUCTION OF THE MODERN IN PORTEAL BETWEEN UNIVERSAL AND SINGULAR. Edite Maria Figueiredo e Rosa • LA SILLA DEL GATEPAC: UN VIAJE COLECTIVO DE IDA Y VUELTA / THE GATEPAC CHAIR: A COLLECTVE JOURNEY THERE AND BACK AGAIN. Maria Villanueva Fernández; Héc. tor Garcí-Diego Villarias $\bullet$ EL ESPACIO INTERMEDIO Y LOS ORíGENES DEL TEAM $X /$ The Space Between and the origins of Team X. Antonio Juárez Chicote; Fernando Rodríguez Ramirez • PENSAMIENTOS COMPARTIDOS. ALDO VAN EYCK, EL GRUPO COBRAYEL ARTE/SHARED THOUGHTS. ALDOVANEYCK, THE COBRA GROUP, AND ART. Esther MayoralCampa •CLAUDE PARENT EN NUEVA FORMA: LA RECEPCIÓN DE ARCHITECTURE PRINCIPE EN ESPAÑA / CLAUDE PARENT AT NUEVA FORMA: THE READING OF ARCHITECTURE PRINCIPEIN SPAIN. Lucía C. Pérez Moreno • CONSTRUYENDO UNA UTOPIE AUTRE IAMAZING ARCHIGRAM!-50 AÑOS DEZOOM!/ZZZZZRRTT//THUD!/ BLAAM!] / BUILDING AUTOPE AUTRE [AMAZING ARCHIGRAM!- 50 YEARS OF ZOOM!/ ZZZZRRTT// THUD:/ BLAAMI]. Luis Miguel Lus Arana • TAN CERCA, TAN LEJOS: ALDO ROSSI Y EL GRUPO 2C. ARQUITECTURA, IDEOLOGÍA Y DISIDENCIAS EN LA BARCELONA DE LOS 70 / SO CLOSE, SO FAR: ALDO ROSSI AND THE2C GROUP. ARCHITECTURE, IDEOLOGY AND DISSENTS IN THE BARCELONA OF THE 70S. Carolina Beatriz García Estévez • RESEÑAS BiBLIOGRÁFICAS• G. ASPLUND, W. GAHN, S. MARKELUUS, G. PAULSSON, E. SUNDAHL, U. AHRÉN: ACCEPTERA. Pablo López Santana

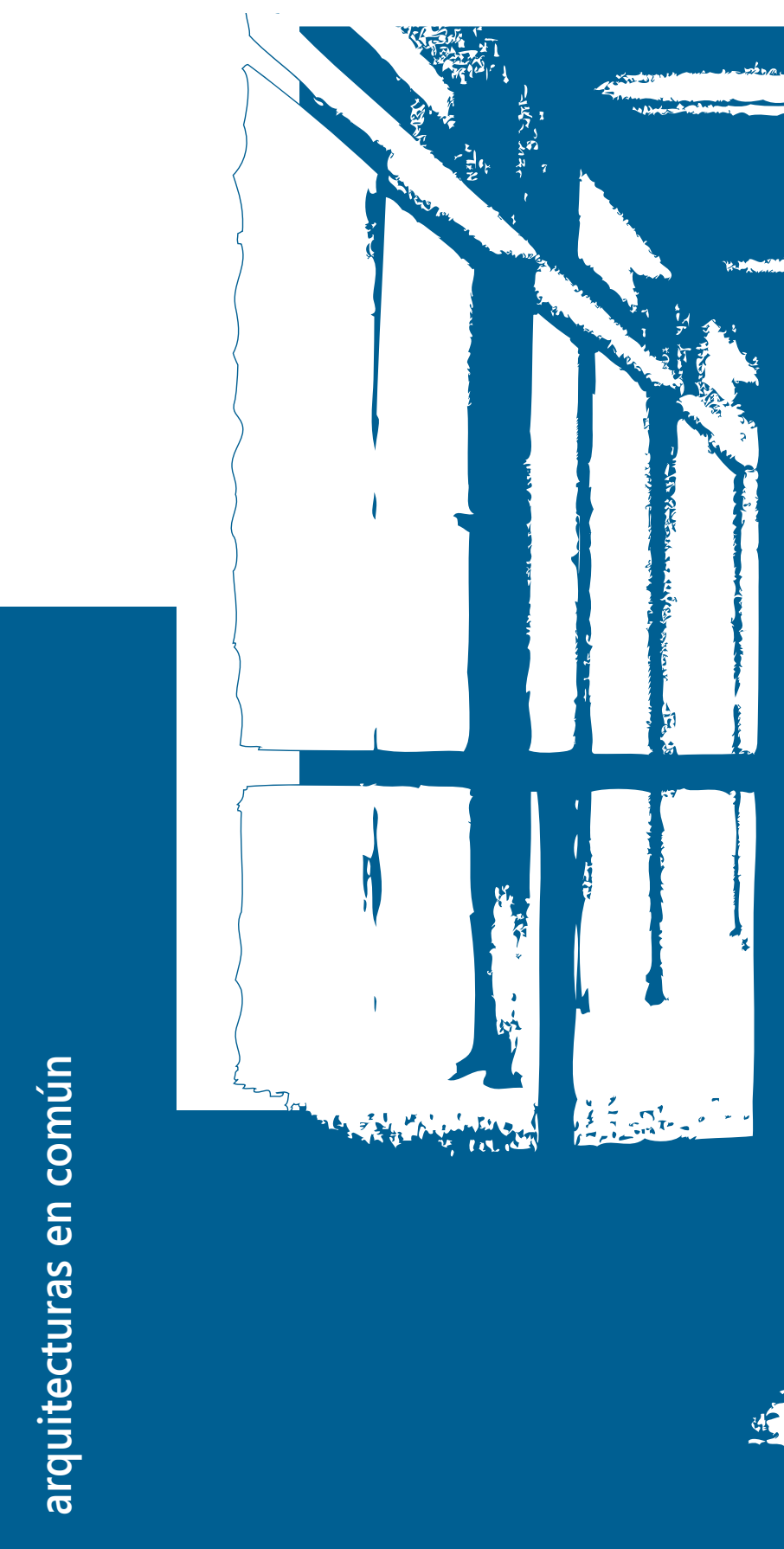

制 20 $t^{\prime \prime 2}$

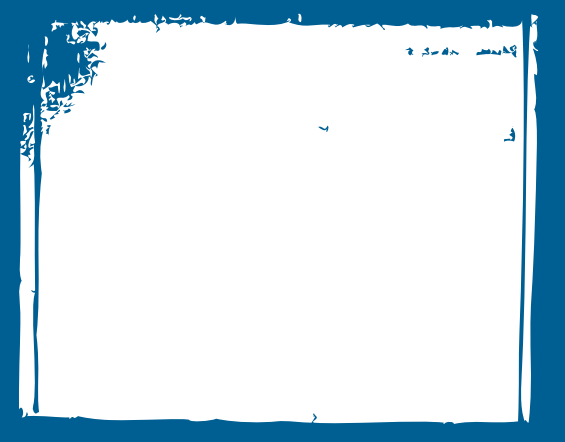

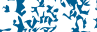

ARQUITECTURAS EN COMÚN

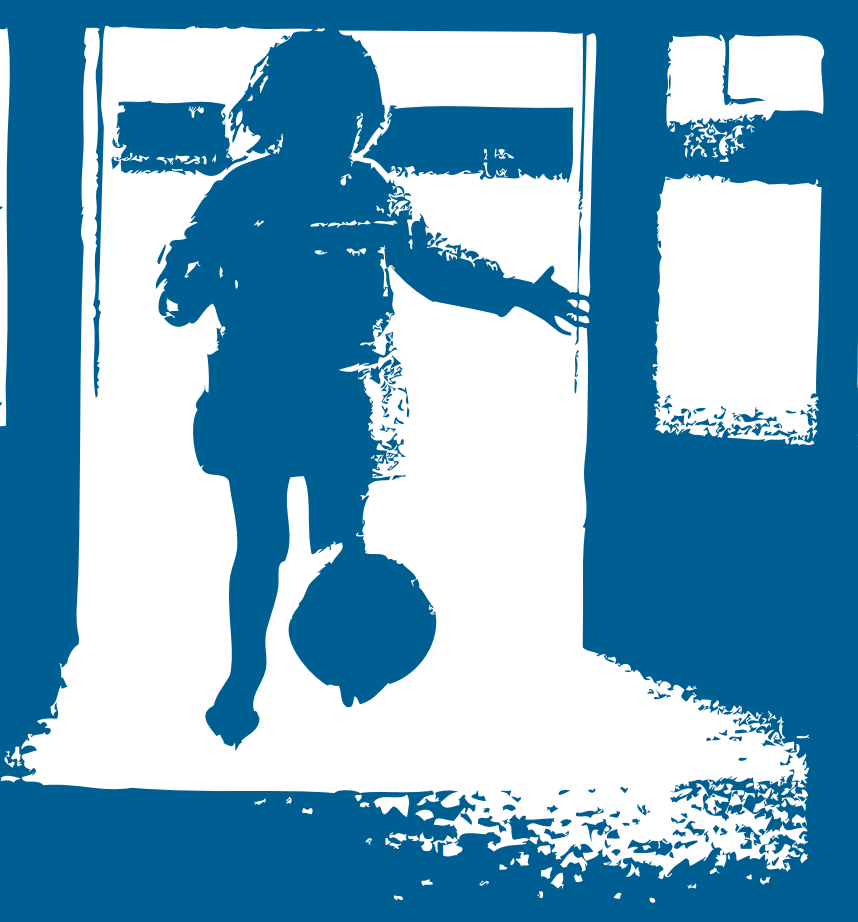

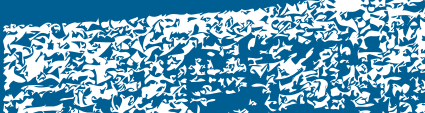

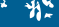
tation 
¿EXISTE UN URBANISMO DEL GATCPAC SIN LE CORBUSIER? / CAN WE TALK ABOUT A GATCPAC

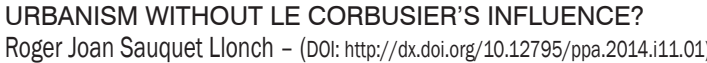

ODAM - A CONSTRUÇÃO DO MODERNO EM PORTUGAL: ENTRE O UNIVERSAL E O SINGULAR I ODAM - THE CONSTRUCTION OF THE MODERN NN PORTUGAL: BETWEEN UNIVERSAL AND SINGULAR

LA SILLA DEL GATEPAC: UN VIAJE COLECTIVO DE IDA Y VUELTA / THE GATEPAC CHAIR: A

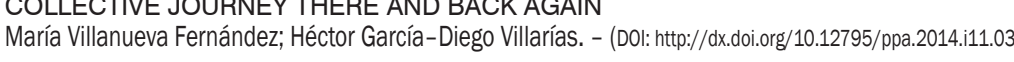

EL ESPACIO INTERMEDIO Y LOS ORÍGENES DEL TEAM X / THE SPACE BETWEEN AND THE

Antonio Juárez Chicote; Fernando Rodríguez Ramírez - (Do: htrp://dx.do.orgrg 10.12795/ppa.2014.11.04)

PENSAMIENTOS COMPARTIDOS. ALDO VAN EYCK, EL GRUPO COBRA Y EL ARTE / SHARED THOUGHTS. ALDO VAN EYCK, THE COBRA GROUP, AND ART

CLAUDE PARENT EN NUIVA FORMA: LA RECEPCIÓN DE ARCHIICTURE PR

PRINCIPE IN SPAIN

CONSTRUYENDO UNA UTOPIE AUTRE [AMAZING ARCHIGRAM! - 50 ANOS DE ZOOM!/ ZZZZRRTT!: THUD!/ BLAAM!] / BUILDING A UTOPIE AUTRE [AMAZING ARCHIGRAM! - 50 YEARS OF ZOOM!/ ZZZZRRTT!/ THUD!/ BLAAMI!

TAN CERCA, TAN LEJOS: ALDO ROSSI Y EL GRUPO 2C. ARQUITECTURA, IDEOLOGÍA Y DISIDENCIAS EN LA BARCELONA DE LOS 70 / SO CLOSE, SO FAR: ALDO ROSSI AND THE $2 C$

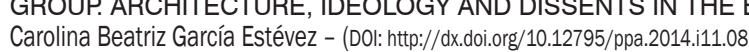




\section{UN FUTURO EN COMÚN}

\section{A FUTURE IN COMMON
Juan José López de la Cruz}

RESUMEN En el contexto actual de crisis e inestabilidad, el modelo en el que los arquitectos han desarrollado hasta ahora su

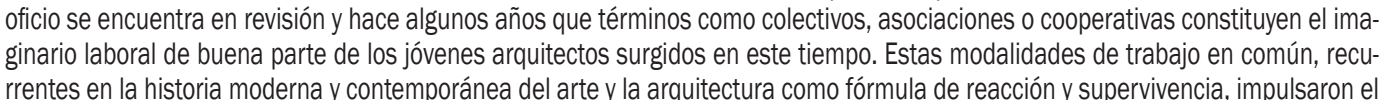
Irentes en la historia moderna y contemporánea del arte y la arquitectura como fórmula de reacción y supervivencia, impulsaron el panorama cultural de sutiempo, actuando como revulsivo crítico ante l l que contemplaban, aunque en ocasiones las expectativas
teóricas apenas tuvieron réplica en la producción constructiva ni impacto en la socieddad. Este número de PPA ofrece un recerrido critico por diversas experiencias que muestra la tendencia a la colectividad de los processos de creación y discusión arquitectónica, con momentos álgidos en determinadas fechas que suelen coincidir con épocas de tránsito cultural. De su lectura podrímos deducir que no hay fớrmula de organización profesional que garantice un mejor resultado que otras, dependiendo en todo caso de la avidez creativay propositiva de quienes las integren, restarían por analizar otros métodos de trabajo cuyas estructuras permanecen
casi siempre al margen de la critica y que parecenn gozar hoy día de las preferencias del mercado bajo el pretendido paradigma de Ia eficaciay ya fiabilildad.

SUMMARY IIn the current context of crisis and instability, the model by which architects have developed their profession until now, is under review. For some years, terms such as collectives, associations or cooperatives, have constituted the working imagination of a good many of the young archintects who have emerged during this time. These modalities of working in common, recurrent in of their time They were acting expectations were scarcely matched by constructive production or impact on society. This edition of PpA offers a critical tour of aiverse experiences which demonstrate the trend towards the collectivity of the processes of architectural creation and discussion, with critical moments on determined dates that usually coincide with times of cultural transition. From its interpretetation, we could cases on the creative and proactive avidity of those who form them they would detract by analysing other working methods whose structures almost always remain beyond, and which seem nowadays to enjoy the preferences of the market under the supposed paradigm of effectiveness and reliability.

KEYWORDS profession; review; processes; common; criticism; future

Persona de contacto / Corresponding author: estudio@sol89.com. Escuela Técnica Superior de Arquitectura. Universidad de Sevilla

as situaciones de inestabilidad económica y social como la presente incitan a repensar tos modelos profesionales que hasta entonces se han considerado válidos. Estas revisiones atañen no sôlo al fin mismo del oficio sino también a las circunstancias y procedimiento desarrollados hasta ese mundo ya superado, tal vez en un deseo acelerado de exorcizar aquello que se ha malogrado y poder sustituirlo cuanto antes. En esta tesitura, el modo en el que los arquitectos desarrollan su tarea no se ha visto libre de este examen de conclencia y hace algunos anos que terminos como colectivos, asociaciones o cooperativas constituyen el inaghario laboral de buena parte de los jovenes arquitectos surgidos en este tiempo. La figura del arquilecto solitario, acodado sobre su mesa cayo lápiz intermedia entre el universo contenido en su cabeza y el mundo retratado en el papel, es sustituida por el anhelo de explorar olras formas de relación protesional, quizás como reacción recurrente de cada generación al modelo que ha heredado, como traducción del espacio de trabajo a las nuevas En esta emergencia colaborativa de principio de siglo han de haber influido sin duda dos circunsEn esta emergencia colaborativa de principio de siglo han de haber inifluido sin duda dos circuns-

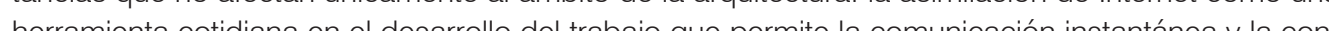
herrán trones alternativos que soslayen la necesidad de intermediarios para progrear y rertir ga situción hereda Amba qurcunntancin la por los más jóvenes quienes no vislumbran un lugar profesional en el mundo ta como fue silgnacio

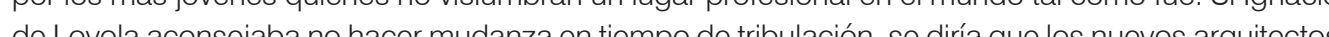
han desódo el consejo del jesuta y se inclinan por cambiar el modelo orgaizativo de la profecín por un traslado literal allá donde sean bien recibidos para poder subsistir. 
Pero ademas de las razones de caracter coyuntural, cabria citar otras de raiz cultural, histórica o como alternativa a la práctica singular de la arquitectura. La avidez intelectual de arquitectos y artistas por compartir sus inquietudes creativas con otros afines ha provocado que a lo largo de la historia estas disciplinas sean ricas en agrupaciones surgidas para poner en común las ideas y tratar de materializarlas. Con el tránsito del conocimiento desde el gremio a la academia, la adhesión a unas ideas y modos de trabajar trascendió de la transmisión paternalista que promete la etimología gremial -regazo-al bullicio de las Escuelas, lugar común de la discusión. No es de extrañar entonces que buena parte de los grupos que protagonizan la historia del arte y la arquitectura surgieran de las aulas o en torno a ellas, consituluidos a partir de la afiliación a una corriente emergente y la consecuente reacción rrente a lo existente. En todo caso, es rasgo común a la mayoría de estos colectivos la juventud de sus miembros, como si en el proceso de maduración de la personalidad propia uno se amparase en la del grupo para afianzar sus ideales y, nuevamente, abrirse camino; valga como ejemplo el llamamiento que el Novembergruppe llevaría a cabo a sus acólitos en su manifiesto fundador de diciembre de 1918: "Dirigimos nuestro saludo fraternal a todos los artistas expresionistas, cubistas y futuristas que se sientan llamados y responsables, deseando que se adhieran a nosotros como socios. Nosotros nos sentimos jóvenes, libres y puros

Entonces como ahora, también en tantas ocasiones a lo largo del siglo XX, las condiciones sociales y la mejora de las comunicaciones favorecieron el surgimiento de multitud de estos grupos de los que el número 11 de PPA da buena cuenta. Es posible atender a esta publicación como una narración ininterrumpida que muestra la tendencia a la colectividad de los procesos de creación y discusión arquitectónica, con momentos álgidos en determinadas fechas que suelen coincidir con épocas de fránsito cultural. Constructivistas, dadaístas, expresionistas y neoplasticistas recorrieron el inicio del siglo XX congregando diversas miradas hacia una nueva era que establecía normas inéditas para el liempo y el espacio a traves de trasvases provenientes de la ciencia; el GATEPAC en España y ODAM en Portugal, al igual que ofros grupos locales agrupados alrededor de los CIAM, Como el Grupo 7 a finales de los años los paises nórdicos, infittrarian la nueva arquitectura moderna en cada latitud, el Team 10 lo fórmu cuchar lean e co Grupo Cón la ln de los compleja y provechosa, la decadá de los sesenta, acaso donde se miran hoy buena parte Archigram y Architecture Principe, trabajeron junto con urbanistas, socílogos o filósofos, editaron

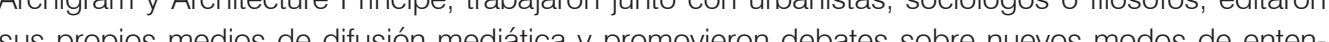
der la disciplina arquitectónica finalmente, Aldo Rossi y Robert Venturi amalgamaron en torno a sus manifiestos los grupos que lidear fin que quedan atestiguados en estas páginas por el periplo del grupo 2C con partidarios en Santiago, Sevilla y Barcelona.

Resta aún por escribir el articulo que dibuje el retrato de lo que está sucediendo en el presente, que tamice la multitud de grupos que pueblan la constelación de asociaciones y plataformas que alumbra el más joven panorama actual de la arquitectura española. Quizás ese texto viniera a corroborar lo que la historia ha relatado en numerosas ocasiones; y es que estas agrupaciones, colectivos o cualquiera que sea el apelativo que evoque la disolución del autor en un conglomerado mayor impulsaron el panorama de la arquitectura y la cultura de su tiempo, actuando como revulsivo critico ante lo que contemplaban, aunque en ocasiones las expectativas teóricas apenas tuvieron réplica en la producción constructiva ni impacto en la sociedad. Se podría afirmar que los procesos de colaboración que pusieron en marcha entre distintas disciplinas y geografias, asi como los medios empleados para la difusión de sus ideas, fueron en muchas ocasiones más poderosos que sus resultados, los más brillantes de los cuales parecian surgir finalmente desde la mirada singular de algunos de sus miembros, alimentando la paradoja de que en muchos casos fue desde estas prácticas en común de donde surgieron buena parte de las más férreas individualidades que protagonizaron la arquitectura y el arte del siglo XX. Dudariamos entonces de si al igual que en aquella máxima que reza que entre todos sabemos todo, la multitud en el proyecto de arquitectura garantiza la excelencia en el resultado o, al menos, si lo hace en mayor medida que otras fórmulas, parece depender en todo caso de la avidez creativa y propositiva de quienes las integren.

Cabría por último permanecer atentos a otros modos de trabajo del arquitecto que quedan fuera de estas lecturas teóricas e historiográficas. Ocupados en disquisiciones sobre las bondades del oficio compartido como alternativa a la experiencia individual o sus carencias frente a los embates del mercado que obliga a estructuras más reconocibles, podriamos olvidar que hoy dia la práctica de la arquitectura, en lo que de común tiene a ambos planteamientos de oficio ético y reflexivo, no está puesta en cuestión por la elección de un modelo $u$ otro, sino por la predilección de parte del mercado por polarizar la profesión bajo el paradigma de la gran oficina que irradia una ilusión de eficacia y profesionalidad donde los departamentos de creación del proyecto son una parte estanca, cas

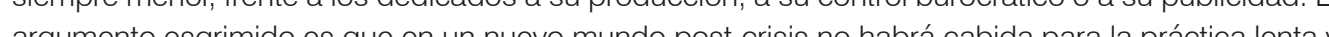
argumento esgrimido es que en un nuevo mundo post-crisis no habrá cabila para la práclica lentay

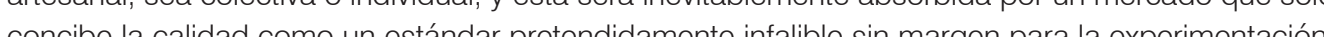
Frente a este razonamiento y los argumentos neoliberales que oculta sorprendentemente asumidos

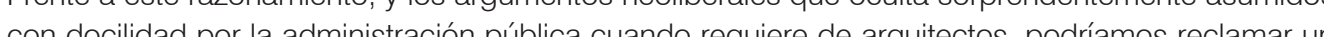
futuro en común de resistencia acaso como la del poeta, el artecano el filósofo o el cientffico, en el que trabajendo a solas o colectivemente participemos del mundo para despús repensalo pacentemente desde nuestras mesas. 